\title{
Downregulation of $h s p 22$ gene expression in Drosophila melanogaster from sites located near chemical plants
}

\author{
L.M. Magdalena ${ }^{1}$, E.C. Coipan ${ }^{2}$, A.F. Vladimirescu², L. Savu', \\ M. Costache ${ }^{4}$ and L. Gavrila ${ }^{1}$ \\ ${ }^{1}$ Department of Genetics, University of Bucharest, Bucharest, Romania \\ ${ }^{2}$ National Institute of Research, \\ Development for Microbiology and Immunology "Cantacuzino", \\ Bucharest, Romania \\ ${ }^{3}$ Genetic Lab SRL, Bucharest, Romania \\ ${ }^{4}$ Department of Biochemistry and Molecular Biology, \\ University of Bucharest, Bucharest, Romania \\ Corresponding author: L.M. Magdalena \\ E-mail: geolamon@yahoo.com / monica@botanic.unibuc.ro
}

Genet. Mol. Res. 11 (1): 739-745 (2012)

Received May 27, 2011

Accepted November 23, 2011

Published March 22, 2012

DOI http://dx.doi.org/10.4238/2012.March.22.4

\begin{abstract}
A common physiological response of organisms to environmental conditions is variation in gene expression, especially true for genes encoding for heat shock proteins. In insects, this process has been examined for induced heat or cold stress. The putative longterm imprinted/acquired heat shock protein response due to unfriendly environmental conditions has been far less studied. The Drosophila melanogaster hsp22 gene, which has been extensively reviewed as being sensitive to different changing life conditions, was examined by qRT-PCR, using carboxy-X-rhodamine. In the present study, we focused on the detection of hsp22 level of transcription in three $D$. melanogaster isolates, collected from sites located near different chemical plants in Romania and subjected to one-year adaptation to laboratory conditions. In all isolates, the $h s p 22$ gene expression
\end{abstract}


was determined using the housekeeping genes Gapdh1 and UbcD10 as internal controls. According to our experimental results, the $D$. melanogaster hsp 22 gene was significantly downregulated compared to the same gene in $\mathrm{w}^{1118}$ iso, used as a calibrator. We showed that hsp 22 could play an important role in relation to stress resistance and adaptation. This study highlights the importance of in vivo studies to demonstrate genome plasticity to overcome different damages induced by any presumed source of stress.

Key words: Drosophila melanogaster; hsp22 downregulation; RT-PCR; Genome plasticity

\section{INTRODUCTION}

Heat shock proteins (Hsp) are considered to play crucial roles in tolerance to environmental stress and in thermal adaptation (Feder and Hofmann, 1999; Frydenberg et al., 2003; Hoffmann et al., 2003; Sørensen et al., 2003). Based on their sequence homology and their dimensions, the Drosophila (Diptera: Drosophilidae) hsp genes have been divided into several families. Their functions include transport, folding, unfolding, assembly/disassembly, and degradation of misfolded or aggregated proteins (Parsell and Lindquist, 1993; Feder and Hofmann, 1999; Sørensen et al., 2003). Heat shock genes can also be defined as being a subset of a larger group of genes coding for molecular chaperones that are involved in housekeeping functions in the cell. Since their molecular tasks are diverse, they are still very much investigated. Many $h s p$ are upregulated in the immediate response to a diverse array of stresses (Feder and Hofmann, 1999). In arthropods, environmental stressors such as heat, heavy metals, ethanol, and desiccation (Hoffmann and Parsons, 1991; Tammariello et al., 1999; Hoffmann et al., 2003) induce them. The possibility of cold stress to activate the heat stress responses has also been investigated (Colinet et al., 2010).

For a long time Hsp22 was thought to be a key player in cell protection against oxidative injuries (Morrow et al., 2004), a typical feature of chilling injury (Rojas and Leopold, 1996). Hsp22 has shown beneficial effects on early-aging events, corroborating the pivotal role of mitochondria in the process of aging. Several implications of Hsp22 in many other different physiological processes are also well documented, such as preserving the integrity of the actin cytoskeleton and microfilaments (Sun and MacRae, 2005), insect cold tolerance (Colinet et al., 2007; Kim and Denlinger, 2009), preventing caspase-dependent apoptosis (Concannon et al., 2003) a process that occurs during heat and cold stress in Drosophila cells (Yi et al., 2007), etc. Quite unexpectedly, Hercus and co-workers (2003) found long-lasting effects on life span in flies that as young adults received mild stress treatments (giving approximately $30 \%$ of maximum $h s p 70$ expression). Consequently, the implication of heat shock proteins in the regulatory mechanisms for adaptation to environmental stress is not entirely known (Lauter and Doebley, 2002; Mitchell-Olds and Knight, 2002). Considering this, we have started to investigate the transcriptional level of $h s p 22$ in three different $D$. melanogaster isolates, collected from sites located near chemical plants in three Romanian counties, after giving the flies some time to become accustomed with the new laboratory living conditions. This kind of investigation has not received too much attention until now. Control D. melanogaster labora- 
tory flies, $\mathrm{w}^{1118}$ iso strain, were processed at the same time as the acclimated flies. Therefore, after approximately one year of accommodation, we investigated in each selected isolate the expression patterns of the $h s p 22$ gene, using quantitative RT-PCR (qRT-PCR). We focused on the investigation of the hsp22 gene, because it is one of the most encountered genes reported to be sensitive to different stress conditions. Although $h s p$ have been found to be implicated in both hot and cold survival, there is little direct evidence in the literature regarding their inherited/acquired genome variation, in the long term.

\section{MATERIAL AND METHODS}

\section{Selection of fruit flies}

We conducted our experiments on three $D$. melanogaster isolates from sites located near different chemical plants that release nitrogen and sulfur compounds: Poli (Policolor region, Bucharest; 44.410885 N, 26.179381 E), Seaca (Teleorman County, 44.154140 N, 24.765083 E), and Brăila (Brăila County, 45.235825 N, 17.938320 E). All fruit flies were collected in September 2008, by trapping (yeast-banana bait). We then generated wild-type laboratory strains starting from one female from each isolate. The fruit flies were maintained under laboratory conditions, at room temperature, on standard cornmeal and agar medium, in $50-\mathrm{mL}$ bottles, for several generations, for approximately 1 year. The study included a control laboratory strain: isogenic $\mathrm{w}^{1118}$ ( $\mathrm{w}^{1118}$ iso, 2150iso, 3150iso; Bloomington stock \#6326), which was maintained under the same laboratory conditions, for one year. Because both sex and age can differentially affect $h s p$ expression (Sørensen et al., 2003), all tests were performed using synchronized 15 - to 25 -day-old males and females, collected in equal numbers $(25+25)$. After rapid selection, minimizing the $\mathrm{CO}_{2}$ exposure, flies were directly transferred to 2-mL screwcap storage tubes, and subjected to mRNA isolation.

\section{Messenger RNA extraction and reverse transcription}

Fruit flies were ground in 1.5-mL tubes. RNA extraction and purification was performed using kits and magnetic stands (Promega kit, PolyATtract System 1000), following manufacturer instructions. Messenger RNA was eluted with $400 \mu \mathrm{L}$ DEPC (diethyl pyrocarbonate)treated water, and 10 and $20 \mu \mathrm{L}$ were then aliquoted into tubes. Next, messenger RNA was quantified using a nanodrop spectrophotometer (ACTGene ASP-2680). Duplicates of highquality RNA samples (i.e., biological replicates) were obtained for each strain. For reverse transcription to cDNA, the Reverse Transcription System 1000 (Promega) was used according to manufacturer instructions. cDNA was quantified and stored at $-20^{\circ} \mathrm{C}$ until use.

\section{Real-time PCR}

Single coding sequence of the heat shock protein 22 (hsp22, CG4460, FBgn0001223) gene of $D$. melanogaster was retrieved from Flybase. The housekeeping genes glyceraldehyde 3 phosphate dehydrogenase 1 (Gapdh1, FBgn0001091) and ubiquitin conjugating enzyme 10 (UbcD10; FBgn0026316) were chosen according to literature recommendations (Edwards et al., 2009; Van Hiel et al., 2009). Moreover, they seemed to exhibit a good mRNA expression 
level in adult Drosophila fly, according to the Drosophila gene expression atlas, which provide a quick answer about the expressed/enriched gene of interest in the adult fly (Chintapalli et al., 2007). The primers used in this experiment were designed using Beacon Designer 7 (PREMIER International Biosoft). The primer sequences are: for $h s p 22$ (forward) 5'-GTCAA CAAGGATGGCTACAA-3' and (reverse) 5'-CACCAGGACAACGCTCTC-3', and the length of the amplicon is $90 \mathrm{bp}$; for Gapdh1 (forward) 5'-CTCCACCGACTTCTTCAG-3' and (reverse) 5'-AGATTAGCTTGACGAACTTATC-3', and the length of the amplicon is $89 \mathrm{bp}$; for UbcD10 (forward) 5'-TTAAGACACGCATCTACC-3' and (reverse) 5'-AGTTCTCCGTACTG ATAATG-3', and the length of the amplicon is $75 \mathrm{bp}$. We performed electronic PCR for all primer pairs on the reference D. melanogaster genome to check for primer specificity (http:// www.ncbi.nlm.nih.gov/tools/primer-blast/), and quality (avoiding primer dimerization and hairpin structures). Real-time PCR was performed on the Abi Prism 7000 System, using the ROX detection channel. Reactions were performed on 96-well plates, using GoTaq qPCR Master Mix (Promega), in a final volume of $20 \mu \mathrm{L}$ including $2 \mu \mathrm{L}$ cDNA sample (containing between 11-12 ng/ $\mu \mathrm{L}$ ), $1.6 \mu \mathrm{L} 10 \mathrm{mM}$ primer mix, and $10 \mu \mathrm{L} 2 \mathrm{X}$ GoTaq qPCR Master Mix (Promega). Supplementary CXR 100X (containing carboxy-X-rhodamine, Promega) solution was added, according to the standard protocol. After $2 \mathrm{~min}$ at $50^{\circ} \mathrm{C}$ and $10 \mathrm{~min}$ at $95^{\circ} \mathrm{C}$, the cycling conditions were as follows: 40 cycles of $95^{\circ} \mathrm{C}$ for $15 \mathrm{~s}$ and $56^{\circ} \mathrm{C}$ for $1 \mathrm{~min}$. The ratio of the target $h s p 22$ gene was expressed in all three samples/isolates: Poli, Seaca and Brăila, versus matched $\mathrm{w}^{1118}$ iso laboratory control strain (calibrator) and normalized using both housekeeping reference genes (Gapdh1 and UbcD10). All reactions were run in triplicate. RT-PCR products were identified on a $3 \mathrm{UV}^{\mathrm{TM}}$ Transilluminator (UVP ${ }^{\circledR}$ Laboratory Products) after electrophoresis on $2.5 \%$ agarose gels and staining with ethidium bromide.

\section{Statistical analysis}

$\Delta \Delta \mathrm{C}_{\mathrm{T}}$ values were calculated using the average $\Delta \mathrm{C}_{\mathrm{T}}$ from each sample. Fold differences were calculated using fold difference of $2^{-\Delta \Delta C}$ (Livak and Schmittgen, 2001). The RQ (relative quantification) logarithmic values were used $\left(\log _{10} R Q\right)$. Difference in gene expression level between RT-PCR runs with the same endogenous control was tested for statistical significance using one-way ANOVA. The mean gene expression levels relative to two different endogenous controls (Gapdhl and $U b c D 10)$ were tested for statistical significance using the Student $t$-test. The differences were considered to be significant for $\mathrm{P}<0.05$.

\section{RESULTS}

As previously noted in D. melanogaster, the increased cold stress period was associated with accumulation of chilling injuries and the time needed for recovery increased (Rako and Hoffmann, 2006). Therefore, we collected the flies from their natural habitat and allowed them to become accustomed to the laboratory conditions, so that they could recover. One year later, we investigated in each selected strain the expression patterns of the $h s p 22$ gene, using qRT-PCR. All qPCR assays yielded specific products (i.e., single amplicons).

In our study, the $h s p 22$ gene expression was found to be significantly downregulated in all strains studied, Poli, Seaca, and Brăila, when compared to the transcriptional level of the $h s p 22$ gene in iso, the control laboratory strain (Figure 1). First, we used Gapdh1 as en- 
dogenous control and reactions were run in triplicate. The gene expression levels were not significantly different between the runs (ANOVA, $\mathrm{P}=0.61$ ).

To validate the results obtained with Gapdh1, we performed a supplementary RT-PCR run using UbcD10 as endogenous control. The $\log _{10} \mathrm{RQ}$ results also indicated a downregulation of $h s p 22$ gene expression. The difference in hsp22 expression levels between the two endogenous controls was not statistically significant (Student $t$-test, two-tailed, $\mathrm{P}=0.98$ ). In both cases, the Brăila isolate expressed hsp22 at the highest level while the Seaca isolate revealed the lowest expression level (Figure 1).
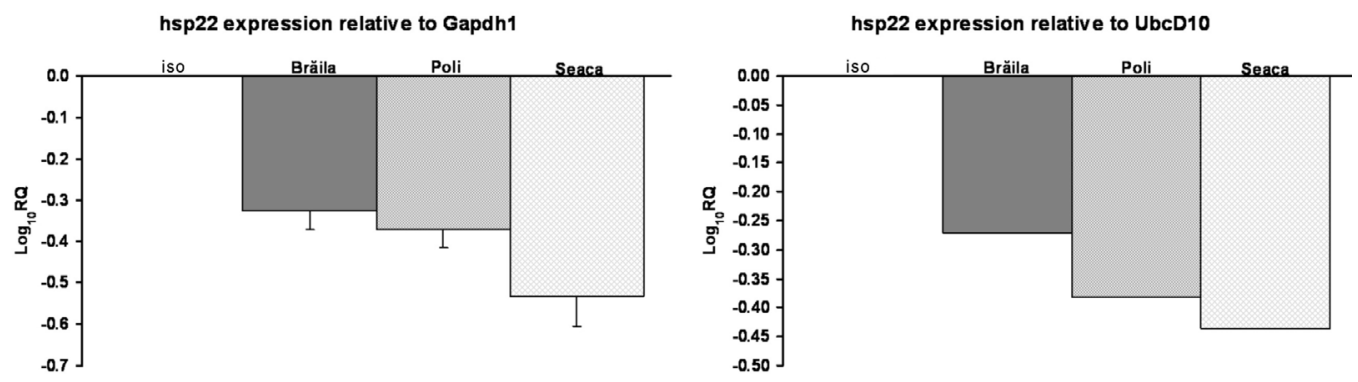

Figure 1. Relative expression of the hsp22 gene in different Drosophila isolates (iso, Brăila, Poli, and Seaca). Error bars denote 1 standard error.

\section{DISCUSSION}

It is known that in arthropods, environmental stressors such as heat, heavy metals, ethanol, and desiccation (Hoffmann and Parsons, 1991; Tammariello et al., 1999; Hoffmann et al., 2003) induce heat shock genes. Previously, it was suggested that stresses of an environmental and/or genetic basis are predicted to affect future populations (Sørensen et al., 2003). Additionally, Hercus and co-workers (2003) found long lasting effects on life span in flies that as young adults received mild stress treatments (giving approximately $30 \%$ of maximum hsp70 expression). However, the potential of heat shock gene response in the mechanisms for adaptation to environmental stress is not completely known (Lauter and Doebley, 2002; Mitchell-Olds and Knight, 2002).

Consequently, our results are not surprising considering the sites from which the fruit flies were collected - near different chemical plants or producer of varnishes and paints. These environmental conditions could have affected them as a cumulative process, even if they were harvested under laboratory conditions over a long period. Although some authors consider that the adaptation in natural Drosophila populations is normal enough to be considered as days or months (Hoffmann et al., 2003), in our case, in all the investigated strains, even one year of adaptation after being in different environmental conditions did not demonstrate the regular transcriptional expression of $h s p 22$.

The downregulation of the $h s p 22$ gene found in this study suggests that the expression level of this gene may be correlated with acquired genome modifications, leading to genomic adaptation to the specific environmental conditions. Our results are in agreement with previous observation that a key feature of the heat shock response is its suppression following res- 
toration of normal environmental conditions (Parsell and Lindquist, 1993) because some hsps can be detrimental to normal growth (Sørensen et al., 2003). Similar results were obtained in different species of Drosophila, when it was shown that (contrary to predictions at that time) expression of another $h s p$ gene, the $h s p 70$, was lower in lines frequently or continuously exposed to severe stress (Bettencourt et al., 1999; Sørensen et al., 1999; Lansing et al., 2000). The authors' interpretation of the data was that the costs of hsp expression in populations frequently exposed to stress outweighed the benefits and that stress adaptation was achieved through some other means. The same pattern was subsequently found in natural populations of Drosophila (Sørensen and Loeschcke, 2002) and in soil invertebrates exposed to heavy metals (Köhler et al., 2000).

Herein, we also suggest that the downregulation of $h s p 22$ found in all the investigated strains may also underlie the maintenance of some degree of genomic tolerance for surviving, as previously suggested by Gong and Golic (2006). This could mean that $h s p 22$ may play an important role in the plasticity of genome adaptation.

In conclusion, we do not intend to assert that the hereditary background of the investigated flies prior to harvesting in the laboratory is a direct cause of the $h s p 22$ down regulation we observed in our study, but that there may be at least an association. The fruit flies could have inherited some molecular damages, even as a regulatory mechanism, which allowed them to cope with different unusual environmental living conditions. It may be that some other genes cooperate for competing with the aggressive nature condition, but this is something that remains to be investigated.

\section{ACKNOWLEDGMENTS}

Research supported by UEFISCDI Romania, IDEAS grant (\#142/2007). We give our special thanks to Dr. J. Szidonya for kindly providing the control laboratory strain (from Drosophila Stock Center, Szeged, Hungary).

\section{Conflict of interest statement}

The authors declare that they have no conflict of interest.

\section{REFERENCES}

Bettencourt BR, Feder ME and Cavicchi S (1999). Experimental evolution of Hsp70 expression and thermotolerance in Drosophila melanogaster. Evolution 53: 484-492.

Chintapalli VR, Wang J and Dow JAT (2007). Using FlyAtlas to identify better Drosophila melanogaster models of human disease. Nat. Genet. 39: 715-720.

Colinet H, Nguyen TT, Cloutier C, Michaud D, et al. (2007). Proteomic profiling of a parasitic wasp exposed to constant and fluctuating cold exposure. Insect Biochem. Mol. Biol. 37: 1177-1188.

Colinet H, Lee SF and Hoffmann A (2010). Temporal expression of heat shock genes during cold stress and recovery from chill coma in adult Drosophila melanogaster. FEBS J. 277: 174-185.

Concannon CG, Gorman AM and Samali A (2003). On the role of Hsp27 in regulating apoptosis. Apoptosis 8: 61-70.

Edwards AC, Zwarts L, Yamamoto A, Callaerts P, et al. (2009). Mutations in many genes affect aggressive behavior in Drosophila melanogaster. BMC Biol. 7: 29.

Feder ME and Hofmann GE (1999). Heat-shock proteins, molecular chaperones, and the stress response: evolutionary and ecological physiology. Annu. Rev. Physiol. 61: 243-282.

Frydenberg J, Hoffmann AA and Loeschcke V (2003). DNA sequence variation and latitudinal associations in hsp23, 
hsp26 and hsp27 from natural populations of Drosophila melanogaster. Mol. Ecol. 12: 2025-2032.

Gong WJ and Golic KG (2006). Loss of Hsp70 in Drosophila is pleiotropic, with effects on thermotolerance, recovery from heat shock and neurodegeneration. Genetics 172: 275-286.

Hercus MJ, Loeschcke V and Rattan SI (2003). Lifespan extension of Drosophila melanogaster through hormesis by repeated mild heat stress. Biogerontology 4: 149-156.

Hoffmann AA and Parsons PA (1991). Evolutionary Genetics and Environmental Stress. Oxford University Press, New York.

Hoffmann AA, Sørensen JG and Loeschcke V (2003). Adaptation of Drosophila to temperature extremes: bringing together quantitative and molecular approaches. J. Therm. Biol. 28: 175-216.

Kim M and Denlinger DL (2009). Decrease in expression of beta-tubulin and microtubule abundance in flight muscles during diapause in adults of Culex pipiens. Insect Mol. Biol. 18: 295-302.

Köhler HR, Zanger M, Eckwert H and Einfeldt I (2000). Selection favours low Hsp70 levels in chronically metal-stressed soil arthropods. J. Evol. Biol. 13: 569-582.

Lansing E, Justesen J and Loeschcke V (2000). Variation in the expression of Hsp70, the major heat-shock protein, and thermotolerance in larval and adult selection lines of Drosophila melanogaster. J. Therm. Biol. 25: 443-450.

Lauter N and Doebley J (2002). Genetic variation for phenotypically invariant traits detected in teosinte: implications for the evolution of novel forms. Genetics 160: 333-342.

Livak KJ and Schmittgen TD (2001). Analysis of relative gene expression data using real-time quantitative PCR and the 2(-Delta Delta C(T)) Method. Methods 25: 402-408.

Mitchell-Olds T and Knight CA (2002). Evolution. Chaperones as buffering agents? Science 296: 2348-2349.

Morrow G, Samson M, Michaud S and Tanguay RM (2004). Overexpression of the small mitochondrial Hsp22 extends Drosophila life span and increases resistance to oxidative stress. FASEB J. 18: 598-599.

Parsell DA and Lindquist S (1993). The function of heat-shock proteins in stress tolerance: degradation and reactivation of damaged proteins. Annu. Rev. Genet. 27: 437-496.

Rako L and Hoffmann AA (2006). Complexity of the cold acclimation response in Drosophila melanogaster. J. Insect Physiol. 52: 94-104.

Rojas RR and Leopold RA (1996). Chilling injury in the housefly: evidence for the role of oxidative stress between pupariation and emergence. Cryobiology 33: 447-458.

Sørensen JG and Loeschcke V (2002). Decreased heat-shock resistance and down-regulation of Hsp70 expression with increasing age in adult Drosophila melanogaster. Funct. Ecol. 16: 379-384.

Sørensen JG, Michalak P, Justesen J and Loeschcke V (1999). Expression of the heat-shock protein HSP70 in Drosophila buzzatii lines selected for thermal resistance. Hereditas 131: 155-164.

Sørensen JG, Kristensen TN and Loeschcke V (2003). The evolutionary and ecological role of heat shock proteins. Ecol. Lett. 6: 1025-1037.

Sun Y and MacRae TH (2005). Small heat shock proteins: molecular structure and chaperone function. Cell Mol. Life Sci. 62: 2460-2476.

Tammariello SP, Rinehart JP and Denlinger DL (1999). Desiccation elicits heat shock protein transcription in the flesh fly, Sarcophaga crassipalpis, but does not enhance tolerance to high or low temperatures. J. Insect Physiol. 45: 933-938.

Van Hiel BM, Van Wielendaele P, Temmerman L and Van Soest S (2009). Identification and validation of housekeeping genes in brains of the desert locust Schistocerca gregaria under different developmental conditions. BMC Mol. Biol. 10: 56.

Yi SX, Moore CW and Lee RE Jr (2007). Rapid cold-hardening protects Drosophila melanogaster from cold-induced apoptosis. Apoptosis 12: 1183-1193. 